\title{
Der Nachlass Paul de Lagarde in den Spezialsammlungen der Niedersächsischen Staats- und Universitätsbibliothek Göttingen
}

\section{Der Kontext: Die Nachlässe und Handschriften der Niedersächsischen Staats- und Universitätsbibliothek Göttingen (SUB)}

Der Nachlass Paul de Lagardes wird innerhalb der SUB Göttingen von der Abteilung Spezialsammlungen und Bestandserhaltung aufbewahrt. Die Bibliothek hat verschiedene Findmittel und Kataloge zur Erleichterung von Recherche und Benutzung erarbeitet und stellt die Bestände unter anderem für Forschung und Lehre in den Räumen des Historischen Bibliotheksgebäudes sowie als Digitalisate

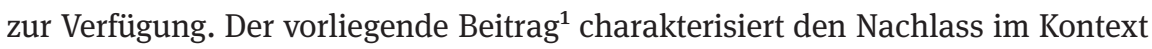
der übrigen Bestände, skizziert den Weg der Bestände von der Aufnahme in die Sammlung über ihre Erschließung, Nutzung bis hin zur Langzeitaufgabe der Digitalisierung und stellt die vorhandenen Findmittel vor.

Die Spezialsammlungen umfassen mehr als 564.000 gedruckte Bücher aus der Zeit bis einschließlich 1900, 318.000 Karten, darunter 65.000 historische Karten und 9.000 Atlanten, mehr als 14.000 Handschriften und Autographen sowie weitere Bestände wie etwa Porträts, Büsten oder Siegel. Nur eine vergleichsweise kleine Zahl abendländischer, mittelalterlicher Handschriften steht deutlich mehr neuzeitlichen Handschriften und nicht zuletzt 2.900 orientalischen Handschriften $^{2}$ gegenüber, die wie beispielsweise ein koptisches Fragment ${ }^{3}$ der

1 Der Beitrag stellt die leicht überarbeitete Fassung des Vortrags von Bärbel Mund und Johannes Mangei dar, der beim Workshop zur wissenschaftsgeschichtlichen Erschließung des Nachlasses von Paul de Lagarde in Göttingen am 15. Januar 2018 gehalten wurde.

$2 \mathrm{Zu}$ den orientalischen Handschriften siehe Haenel, Klaus: Die Handschriftenabteilung. In: Kind-Doerne, Christiane: Die Niedersächsische Staats- und Universitätsbibliothek Göttingen. Ihre Bestände und Einrichtungen in Geschichte und Gegenwart. Wiesbaden 1986. S. 112-129, besonders S. $117 \mathrm{f}$.

3 Edition: Pietschmann, Richard (Hrsg.): Apophthegmata patrum boheirisch. In: Nachrichten der Königlichen Gesellschaft der Wissenschaften zu Göttingen. Phil.-Hist. Kl. 1899, H. 1. S. 36-48. 
Apophthegmata patrum in bohairischer Sprache aus dem 9. oder 10. Jahrhundert ( $2^{\circ}$ Cod. Ms. Lagarde 138 A Cim.) zum Teil direkte Bezüge zu Paul de Lagarde aufweisen. Außerdem befinden sich 412 Nachlässe (Stand: Dezember 2018) aller Fachrichtungen in der Sammlung. Im engeren fachlichen Umfeld der Papiere von Paul de Lagarde sind 32 weitere Nachlässe zur Orientalistik und 35 theologische Nachlässe zu nennen. Insgesamt finden sich in der Sammlung weit überwiegend wissenschaftliche Nachlässe gegenüber nur vergleichsweise wenigen literarischen Kollektionen. Bis auf wenige Ausnahmen wird mit Briefen, Manuskripten und anderen Originalquellen nur unpubliziertes Material übernommen. Dagegen finden sich in der Regel keine Nachlass-Bibliotheken im Bestand. Eine Ausnahme ist die Bibliothek von Carl Friedrich Gauß.4 Lagardes Büchersammlung gehört deshalb auch nicht zu den Beständen der Göttinger Bibliothek; die Bücher wurden auf seinen eigenen Wunsch hin in die USA, an die Universität von New York verkauft. ${ }^{5}$

\section{Der Nachlass Paul de Lagarde}

Der Nachlass Paul de Lagarde umfasst achtzehn laufende Regalmeter und ist damit einer der größeren Nachlässe in der Sammlung. Er besteht aus drei Teilen: dem Hauptteil, der sehr umfangreichen ersten Ergänzung (ehemals als „unsignierter Teil“ bezeichnet) sowie aus später hinzu gekommenen Einzelstücken verschiedener Provenienz.

Die Nachlässe sind in einem besonders gesicherten Magazin untergebracht, das gute konservatorische Bedingungen bietet. Sie sind dort alphabetisch nach den Namen der Nachlasser aufgestellt. Die Papiere Lagardes liegen daher zwischen den Nachlässen des Ingenieurs Hans Georg Küssner (1900-1984) und des Skandinavisten Wolfgang Lange (1915-1984); gegenüber befindet sich der Nachlass Georg Christoph Lichtenberg (1742-1799).

\section{Hauptteil}

Der als „Hauptteil“ des Nachlasses bezeichnete Bestand ist zwischen 1892 und 1894 in die Universitätsbibliothek gekommen. Die Spanne ergibt sich aus dem

4 Dazu Haenel, Handschriftenabteilung, S. $125 \mathrm{f}$.

5 Stern, Fritz Richard: Kulturpessimismus als politische Gefahr. Eine Analyse nationaler Ideologie in Deutschland. Stuttgart 2005. S. 55 (Anmerkung). 

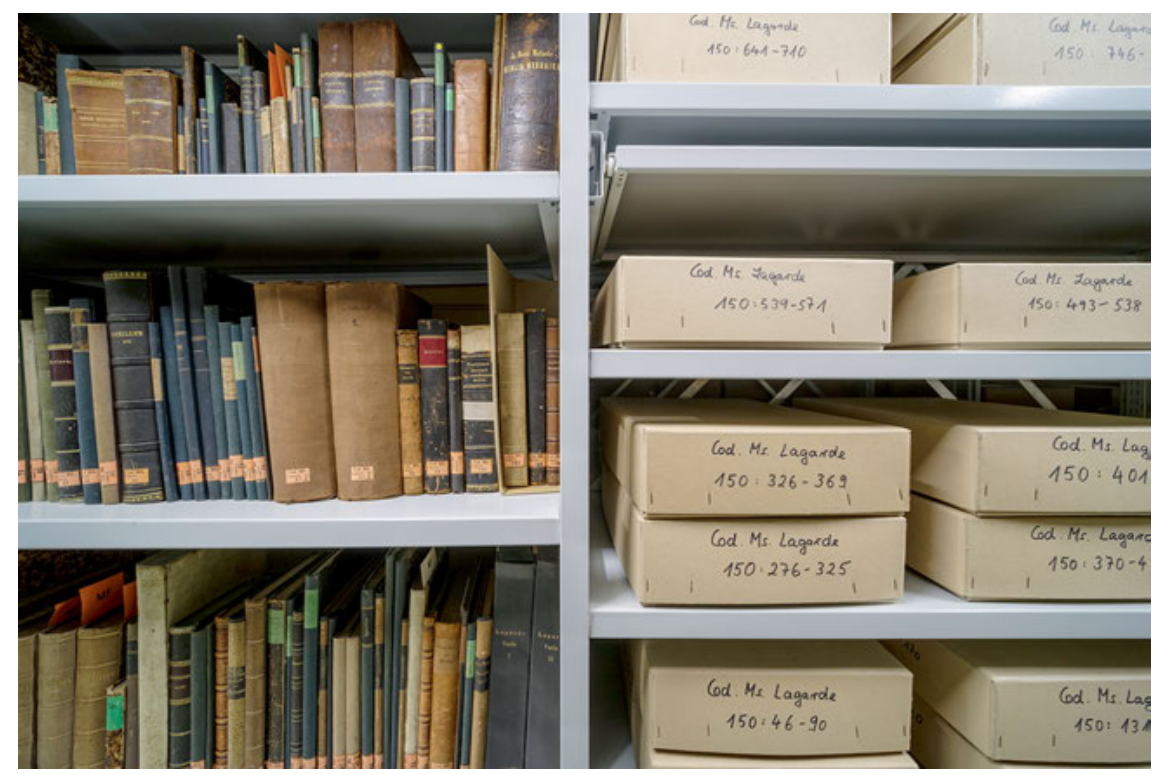

Abb. 1: Nachlassbestand Paul de Lagarde im Magazin der SUB Göttingen () CC-BY-SA 4.0 SUB Göttingen / Martin Liebetruth

Todesdatum Lagardes im Dezember 1891 und dem Erscheinen des dritten Bands von Meyers Katalog 1894, der den Zugang schon verzeichnet. Der Hauptteil umfasst hauptsächlich Handexemplare von Lagardes Publikationen, Manuskripte und orientalische Handschriften und ist nach Sprachen geordnet. Dieser Teil des Nachlasses wird im gedruckten Göttinger Handschriftenkatalog von Wilhelm Meyer in insgesamt 149 Einträgen beschrieben. Dabei umfasst die im Druck erschienene Version 138 Nummern, ${ }^{6}$ dazu kommen elf handschriftlich ergänzte Einträge im Lesesaalexemplar. $\mathrm{Zu}$ Beginn dieses Katalogeintrags dokumentiert Meyer die Bestimmung, dass die Stücke „nur in den Räumen der Bibliothek benutzt werden dürfe[n]“.7 Diese Regelung geht auf die Bestimmungen zurück, die Lagarde am 15. Dezember 1891 selbst im zweiten Nachtrag zu seinem ursprünglich 1886 verfassten „Letzten Willen“ kurz vor seiner Krebsoperation getroffen hat:

6 Meyer, Wilhelm: Die Handschriften in Göttingen. 3 Bde. Berlin 1893-1894, hier Bd. 3, Berlin 1894. S. $133-153$.

7 Meyer, Handschriften, Bd. 3 (wie Anm. 6), S. 133.Vgl. dazu auch den Beitrag „Paul de Lagarde“. In: Paul de Lagarde und die syrische Kirchengeschichte. Hrsg. vom Göttinger Arbeitskreis für syrische Kirchengeschichte. Göttingen 1968. S. 3-7, hier S. 5 f. 
Alle meine wissenschaftlichen Manuscripte, die im Schranke stehen, und etwa verzettelte andere sollen [...] gebunden werden, und danach, ohne dass meine Wittwe zur Eile gedrängt werden darf, der Göttinger Universitätsbibliothek zu freier Benutzung für Jedermann überwiesen werden, mit der Massgabe jedoch, dass sie nirgends hin verschickt, und nur in den Räumen der Göttinger Bibliothek benutzt werden dürfen. ${ }^{8}$

Nur eine Woche später, am 22. Dezember 1891 starb er im Göttinger MariahilfKrankenhaus.

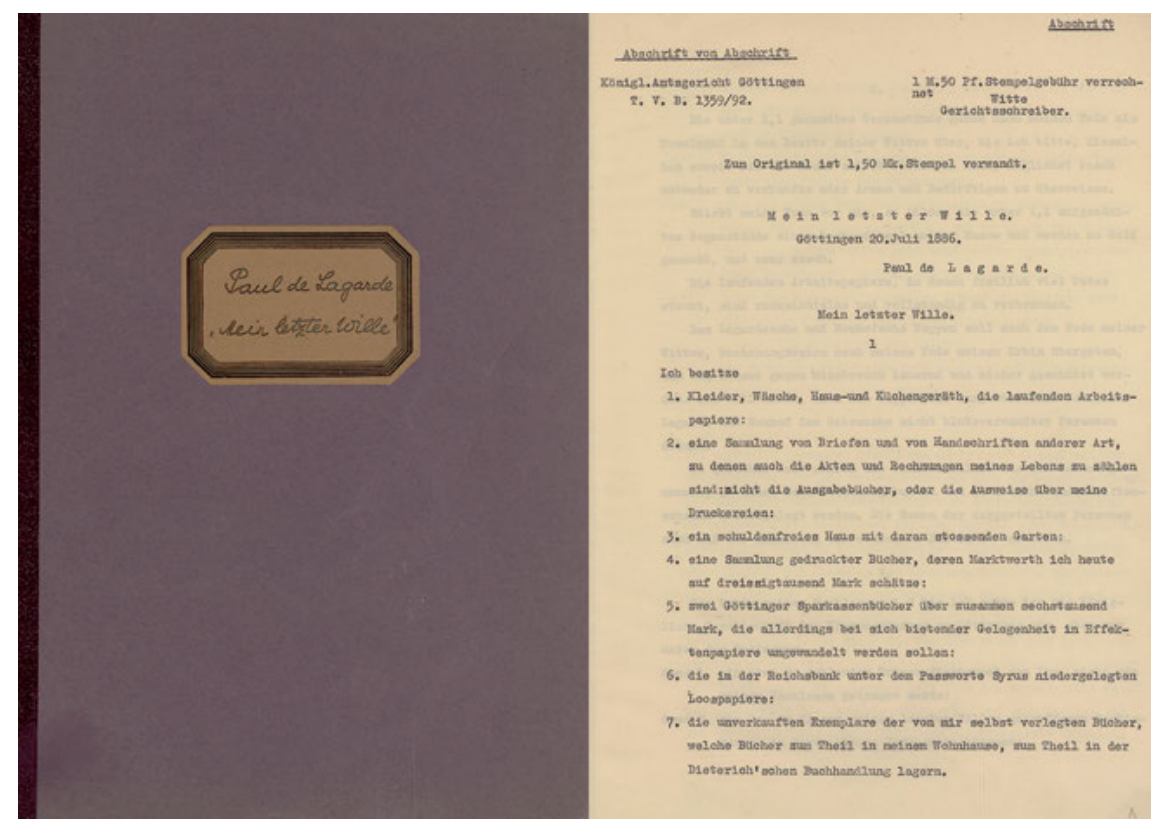

Abb. 2: Zeitgenössische Abschrift des Testaments „Mein letzter Wille“ (c) CC-BY-SA 4.0 Public Domain

\section{Ehemals unsignierter Teil}

In demselben Nachtrag finden sich außerdem Bestimmungen zu Lagardes Briefen, Akten und Rechnungsbüchern:

8 SUB Göttingen, Paul de Lagarde: Mein letzter Wille. Abschrift, Signatur Cod. Ms. Lagarde 187:1, S. $21 \mathrm{f}$. 
Ich bestimme darum, dass alle meine Briefe, Akten, Rechnungsbücher, und alle meine laufenden Arbeiten in den Händen meiner Wittwe verbleiben sollen, mit der Maßgabe, dass diese meine Wittwe der Universitätsbibliothek zu Göttingen Quittung über die dieser Bibliothek vermachten Theile der eben genannten Masse ertheile. Was meine Wittwe der Bibliothek nach gemachtem Gebrauche übergeben wird, ist bis $1925 \mathrm{zu}$ sekretieren. ${ }^{9}$

Dieser Teil befindet sich seit 1901 in der Universitätsbibliothek, durfte aber bestimmungsgemäß erst ab 1925 genutzt werden. Er wurde „am 23. April 1901 in die Bibliothek gebracht [...]“ und ,am 24. [April] durch Herrn G. R. R. Professor Dr. E. Ehlers als Vertreter der Erbin und Oberbibliothekar Dr. E. Lutz für die Bibliotheksverwaltung nach dem hier abgebildeten Verzeichnis (Abb. 3, S. 28) verglichen." ${ }^{10}$

Der ehemals unsignierte Teil wurde 1984 von Brigitte Laue-Eymess (19582011) verzeichnet. Sie erstellte eine maschinenschriftliche Liste von sieben Seiten, die als Grundlage für die Benutzung bis 2003 maßgeblich blieb. ${ }^{11}$

Briefe bilden das größte Segment im unsignierten Teil. Die inzwischen alphabetisch geordnete ,allgemeine Korrespondenz“ Lagardes umfasst 1335 Nummern. Es handelt sich um Briefwechsel mit Kollegen, Schülern, Freunden, Verwandten, Verlegern und Bibliothekaren. Diese Briefsammlung zeichnet sich auch dadurch aus, dass sie viele Briefentwürfe Paul de Lagardes enthält sowie zahlreiche Abschriften seiner Briefe von der Hand seiner Ehefrau Anna. ${ }^{12}$

Wie im Nachtrag zum Testament festgelegt, waren die Briefe jahrgangsweise gebunden worden. Die Witwe Anna de Lagarde hatte jedem der 28 Briefbände ein Korrespondentenregister beigefügt. Anhand dieser Indizes legte der Bibliothekar Johannes Joachim (1864-1949) eine alphabetisch geordnete Korrespondentenkartei an. Die Nutzung des unsignierten Teils seit 1925 konzentrierte sich auf das Briefsegment. Die Korrespondentenkartei ermöglichte das rasche Auffinden von Briefen bestimmter Personen.

Aus konservatorischen Gründen und zur besseren Handhabung wurden die Briefbände 2004 in der Restaurierungswerkstatt der SUB Göttingen fachgerecht aufgetrennt. Die von Anna de Lagarde angelegten Indizes wurden separat abge-

9 Lagarde, Letzter Wille (wie Anm. 8), hier S. 18.

10 SUB Göttingen, Verzeichnis des in besonderem Schranke bis 1925 unter Siegel auf der Königlichen Universitäts-Bibliothek zu Göttingen aufzubewahrenden litterarischen Nachlasses des verstorbenen G. R. R. Professors Dr. Paul von Lagarde“, Signatur Cod. Ms. Bibl.-Arch. B 15 c, Deposita.

11 Sie wird als Anhang 1 zu dem unten näher beschriebenen Findbuch weiterhin dokumentiert. 12 Zur Bedeutung Anna de Lagardes vgl. Sieg, Ulrich: Deutschlands Prophet. Paul de Lagarde und die Ursprünge des modernen Antisemitismus. München 2007. S. 113-133. 


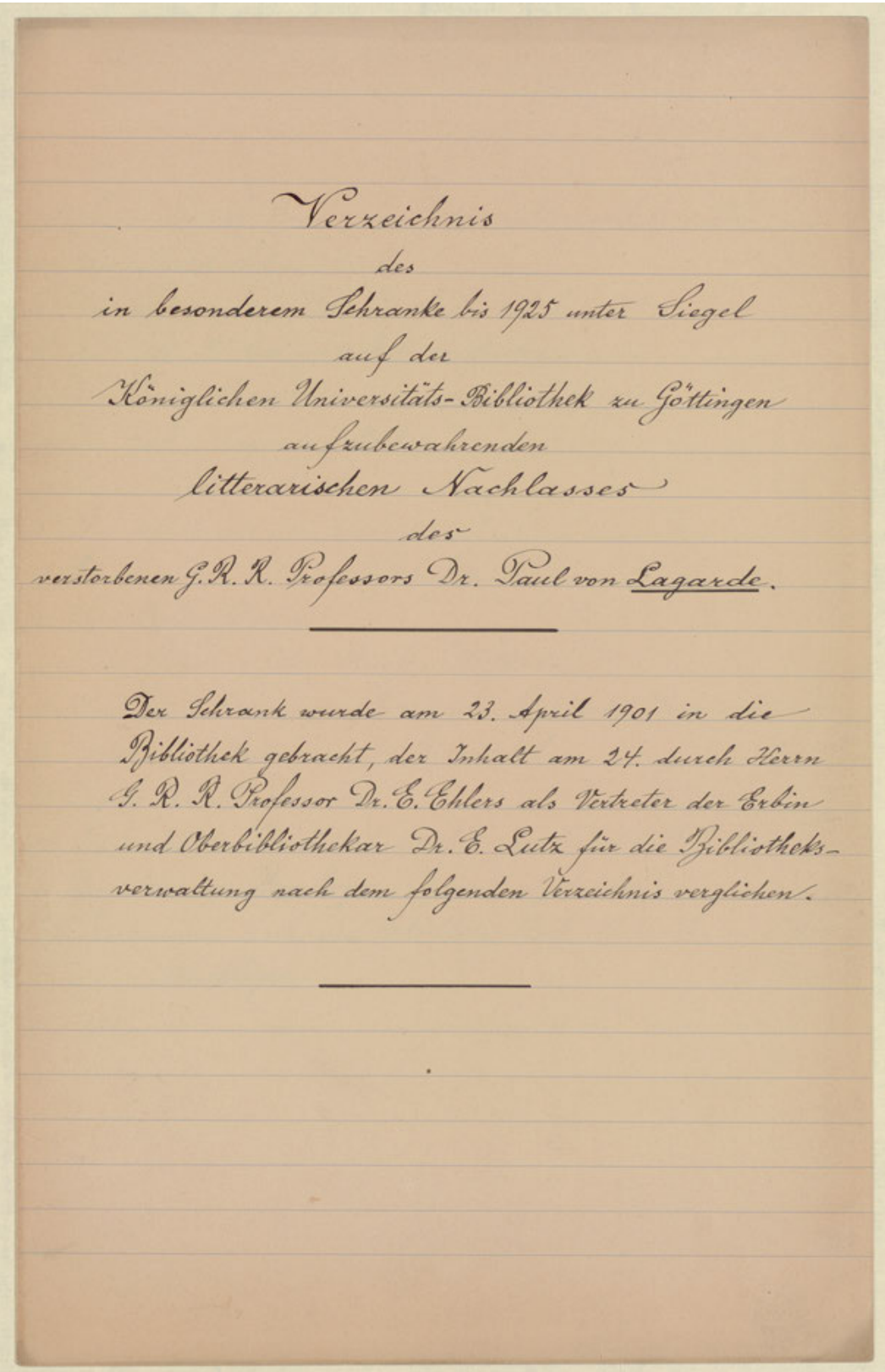

Abb. 3: Titelseite des Verzeichnisses von 1901 @ C CC-BY-SA 4.0 Public Domain 
legt. ${ }^{13}$ Mit Hilfe dieser Indizes kann die Chronologie der Gesamtkorrespondenz rekonstruiert werden. Die Korrespondentenkartei ermöglichte nach dem Auflösen der Briefbände eine rasche alphabetische Sortierung der Briefe.

Anna de Lagarde hatte als Nachlassverwalterin leider eine Reihe von Briefen aus den Bänden entfernt. Zuweilen sind noch Reste dieser Schriftstücke enthalten, wie hier im Falle eines Briefes von Georg Steindorff (Abb. 4, S. 40) ${ }^{14}$.

In der Korrespondentenkartei wurden auch zurückbehaltene und vernichtete Briefe nachgewiesen. ${ }^{15}$ Diese Belege sind erhalten. ${ }^{16}$ Die übrigen Karten aus der Korrespondentenkartei wurden makuliert, weil die auf ihnen vermerkten Informationen bei der Neukatalogisierung in eine Datenbank übernommen wurden.

Zum ehemals unsignierten Teil gehören des Weiteren die mit 416 Nummern sehr umfangreiche Korrespondenz der Witwe Anna de Lagarde sowie Briefe an den Vater Wilhelm Boetticher. Außerdem sind hier Akten, Manuskripte und Notizen, Rezensionen, Zeitungsausschnitte und Varia zu finden. Auch persönliche Dokumente fehlen nicht; dazu gehören u.a. Fotos und Urkunden sowie ein Stammbuch aus Paul de Lagardes Studienzeit. Selbst Alltagsgegenstände (wie eine Uhrkette und ein Petschaft) befinden sich in diesem Nachlass-Segment.

\section{Zur Erschließung der Göttinger Lagarde-Bestände}

Die erwähnten Findmittel für den ehemals unsignierten Teil (Liste nebst Korrespondentenkartei) wurden ab 2003 durch die Katalogisierung unter formalen Gesichtspunkten in der Allegro-basierten Online-Datenbank HANS ${ }^{17}$ abgelöst. Eine Recherche war dort mittels Registern oder durch eine gezielte Suche (nach nur einem Begriff oder in Kombination mehrerer Begriffe) möglich. Nach Abschluss der Katalogisierung 2004 wurde ein Findbuch erstellt. Auf der Startseite der HANS-Datenbank wird dieses Findbuch in leicht überarbeiteter Fassung als PDF-Datei angeboten. ${ }^{18}$ Diese Datei enthält auch die Beschreibung des Hauptteils des Nachlasses aus dem Handschriftenkatalog von Wilhelm Meyer.

13 Sie befinden sich im Nachlass, SUB Göttingen, Signatur: Cod. Ms. Lagarde 150 : Beil. 1.

14 SUB Göttingen, Signatur: Cod. Ms. Lagarde 150 : 1160 / Beil.

15 Zur Rückgabe bzw. Vernichtung von Briefen vgl. SUB Göttingen, Signatur: Cod. Ms. Lagarde 150 : Beil. 2.

16 Sie sind enthalten in: SUB Göttingen, Signatur: Cod. Ms. Lagarde 150 : Beil. 2.

17 Das Akronym HANS steht für „Handschriften, Autographen, Nachlässe, Sonderbestände“. 18 Nachlass Paul de Lagarde. Theologe und Orientalist 2.11.1827-22.12.1891. http://hans.sub. uni-goettingen.de/nachlaesse/Lagarde.pdf (02.12.2018). 


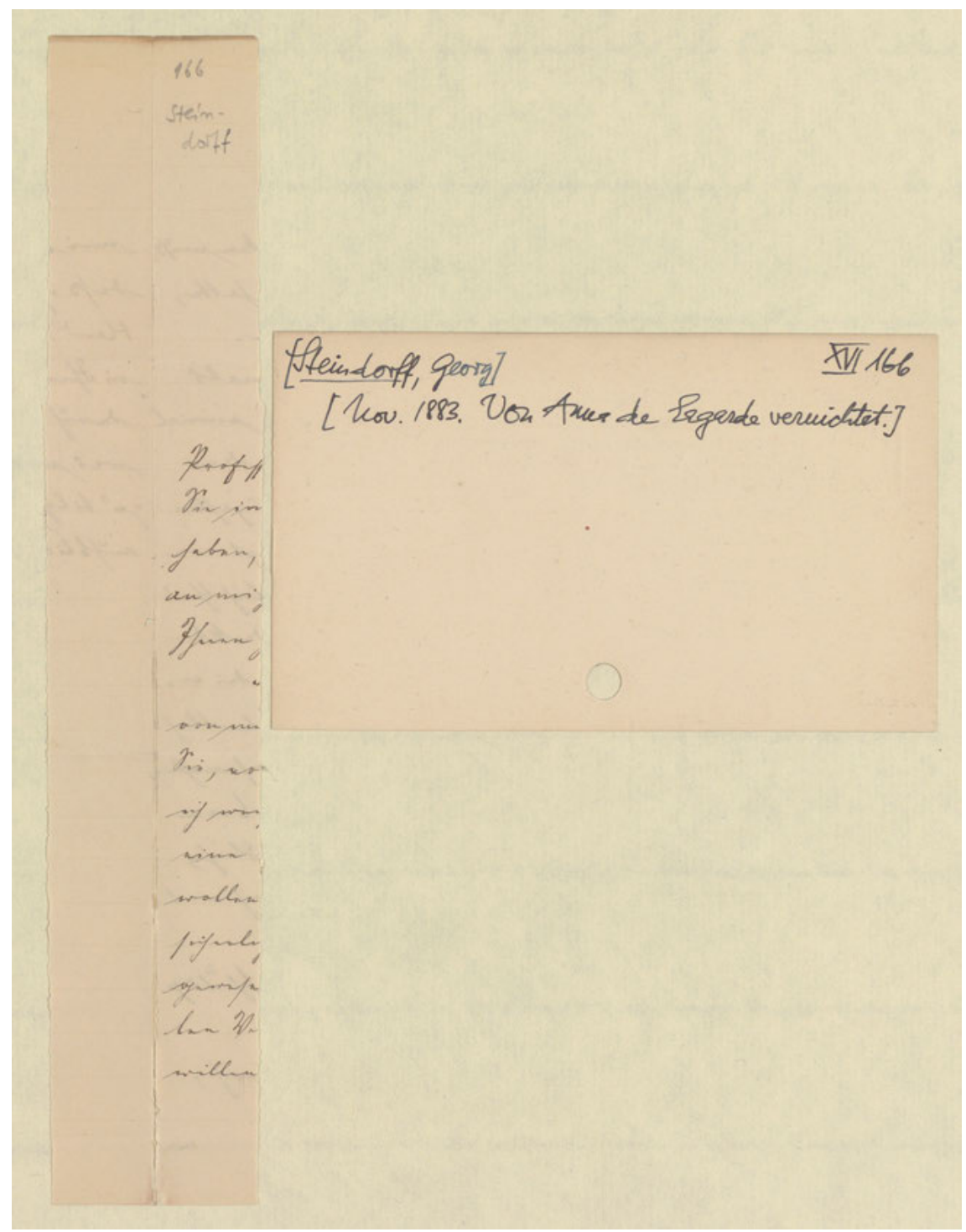

Abb. 4: Reste eines durch Anna de Lagarde entfernten Briefs von Georg Steindorff (C) CC-BY-SA 4.0 Public Domain

Im Jahr 2016 wurden die Erschließungsdaten aus der Datenbank HANS schließlich in das Portal Kalliope migriert; dieses frei im Internet verfügbare 
Portal stellt die Katalogdaten von mehr als 950 Institutionen zur Verfügung. ${ }^{19}$ Dadurch sind grundsätzlich nicht nur die Bestände eines Hauses, sondern die Bestände aller beteiligten Einrichtungen recherchierbar, was besonders bei heute verstreuten Provenienzen von Bedeutung ist. Zu dem Suchbegriff „Paul Lagarde“ finden sich 2041 Treffer (Stand: Dezember 2018), davon beziehen sich mit 1828 die meisten auf Bestände der SUB Göttingen. Weitere Einträge stammen aus der Universitätsbibliothek Leipzig, dem Deutschen Literaturarchiv Marbach, der Bayerischen Staatsbibliothek München, der Württembergischen Landesbibliothek Stuttgart und andere mehr. Kalliope erlaubt ein nachträgliches Eingrenzen der Treffermengen durch Facetten, zum Beispiel durch die bestandshaltende Institution oder auch Urheber, Adressaten, Orte, Gattungen, Sprache und zahlreiche weitere Kriterien. In Kalliope ist außerdem ein virtuelles Findbuch generierbar, dessen Sortierung derzeit allerdings noch nicht stringent realisiert wird.

\section{Digitalisierung}

Die bisher vorhandenen Digitalisate der SUB Göttingen mit Bezug zu Lagarde sind nicht systematisch erstellt worden, sondern gingen vor allem aus der Auftragsdigitalisierung Dritter und aus internen Geschäftsgängen hervor. Diese Digitalisate sind bisher größtenteils noch nicht online verfügbar. Ein Beispiel dafür ist die bereits erwähnte, zeitgenössische Abschrift von Lagardes Testament „Mein letzter Wille“. ${ }^{20}$ Die SUB ist fortdauernd darum bemüht, Teile des Nachlasses, einschließlich einer Reihe von Digitalisaten, vermehrt online zugänglich werden zu lassen. Erste Stücke wie der vierseitige Brief des italienischen Sprachwissenschaftlers und Orientalisten Emilio Teza (1831-1912) an Paul de Lagarde, abgeschickt in Pisa am 25. März 1882, sind bereits online. ${ }^{21}$ Darüber hinaus sind allerdings schon zahlreiche Drucke von Lagarde bzw. über ihn verfügbar. Sie werden über die Präsentation des Göttinger Digitalisierungszentrums (GDZ) bereitgestellt. Unter den Digitalisaten befinden sich etwa der gedruckte Katalog seiner Bibliothek, den seine Witwe Anna de Lagarde im Jahr 1892 vor dem Verkauf

19 Der Kalliope-Verbund. http://kalliope.staatsbibliothek-berlin.de/de/ueber-kalliope/historie.html (02.12.2018).

20 Siehe oben, Abschnitt 4.

21 SUB Göttingen, Signatur: Cod. Ms. Lagarde 150 : 1195. Digitalisat: https://gdz.sub.uni-goettingen.de/id/DE-611-HS-3333389 (02.12.2018). Außerdem sind noch u.a. ein Brief von Hugo Schuchardt (http://resolver.sub.uni-goettingen.de/purl?DE-611-HS-3172690, 02.12.2018) sowie ein Brief an Conrad Kessler (http://resolver.sub.uni-goettingen.de/purl?DE-611-HS-3172668, 02.12. 2018) bereits verfügbar. 
der Bücher publizierte, ${ }^{22}$ oder seine 1874 in der Dieterichschen Verlagsbuchhandlung erschienen „Politischen Aufsätze“.23 Weitere Digitalisate bieten andere Bibliotheken wie z. B. die Bayerische Staatsbibliothek München online an, so etwa Paul de Lagardes Titus-Bostrensis-Ausgabe von 1859. ${ }^{24}$

\section{Schluss}

Der Nachlass von Paul de Lagarde in den Spezialsammlungen der SUB Göttingen stellt weiterhin eine bisher nur zum Teil ausgewertete Quelle mit noch zahlreichen Ansatzpunkten für die Forschung dar. Die verbesserte online verfügbare Katalogisierung der letzten Jahre mag einen ersten Schritt zur Erleichterung solcher Studien darstellen. Die sukzessive Bereitstellung wichtiger Teile des Nachlasses zusammen mit Veröffentlichungen von und über Lagarde als frei verfügbare Online-Digitalisate sind die nächsten Schritte. Doch auch damit wird der Bedarf an erschließenden Arbeiten nicht gedeckt sein. $\mathrm{Zu}$ den digitalen Bildern werden Volltexte und für die Volltexte Auszeichnungen und Verlinkungen sowie digitale Editionen im Sinne des Linked-Open-Data-Ansatzes unerlässlich sein. Die SUB Göttingen ist im Rahmen ihrer Möglichkeiten bestrebt, ihre Beiträge dazu zu leisten.

22 Lagarde, Anna de: Katalog der Bibliothek Paul de Lagarde’s. Zur Erinnerung an den verstorbenen Besitzer überreicht von Anna de Lagarde. Göttingen 1892. Digitalisat https://gdz.sub.uni-goettingen.de/id/PPN550074570 (02.12.2018).

23 Lagarde, Paul de: Politische Aufsätze von Paul de Lagarde. Göttingen 1874. Digitalisat https://gdz.sub.uni-goettingen.de/id/PPN555573346 (13.02.2018).

24 Lagarde, Paul de (Hrsg.): Titus Bostrensis: Titi Bostreni quae ex opere contra Manichaeos edito in codice Hamburgensi servata sunt. Graece. E recognitione Pauli Antonii de Lagarde. Berlin 1859. Digitalisat der Bayerischen Staatsbibliothek http://reader.digitale-sammlungen.de/resolve/display/bsb10606584.html (02.12. 2018). 